\title{
Considerações sobre a CASP-19 no Brasil
}

\author{
Thoughts on CASP-19 in Brazil
}

Consideraciones sobre la CASP-19 en Brasil

Fabia Lima 1
Kátia Patribu 1
Jerson Laks 2
Marilia Siqueira Campos 1

doi: 10.1590/0102-311X00070619
Prezadas Editoras,

O estudo de validação da CASP-19 de Neri et al. 1, publicado em Cadernos de Saúde Pública, é de grande valor, pois analisou uma amostra numericamente significativa de 368 idosos residentes em três regiões do Brasil (Sudeste, Sul e Nordeste).

No entanto, algumas considerações merecem ser discutidas:

(1) O nosso grupo realizou a primeira validação da CASP-19 no Brasil, tendo sido cumpridas todas as etapas para uma boa validação 2. Essa validação está disponível no site da CASP-19 como a versão oficial para uso no português do Brasil (https://casp19.com).

Em relação às etapas de validação, foram traduzidas por tradutores nativos do país de origem da escala e brasileiros bilíngues, tendo sido utilizado o protocolo de Beaton et al. 3 .

Foi aplicada a uma amostra de 87 idosos ( $\mu=75,6 \pm 0,7$ anos) residentes na cidade do Recife, Pernambuco, Brasil, com idade igual ou superior a 65 anos e pertencentes às classes sociais alta, média e baixa renda 2 .

Houve autorização do autor principal da escala (Martin Hyde) e acompanhamento nas etapas da validação.

A análise estatística foi definida e realizada pelo autor principal da escala e um estatístico de seu grupo (Holendro Singh Chungkham).

(2) Após as análises das propriedades psicométricas, a CASP-19 foi reduzida a uma versão menor para ser utilizada no Brasil, a CASP-16, sem perdas quando comparada à versão da CASP-19 2.

Na nossa validação, o domínio Prazer apresentou o menor coeficiente alfa, o que significa que pode ser o domínio que menos contribuiu para a escala, possivelmente, por esta razão. Foram discutidas várias razões possíveis para esses resultados, sobretudo em termos culturais, cujo prazer pode ter sido interpretado pelos idosos brasileiros como um dos significados de satisfação. Essa discussão está no artigo original.

(3) Há uma tendência internacional para que as escalas sejam reduzidas, como uma alternativa de mais rápida aplicação ao instrumento, como exemplo, a escala padrão ouro de qualidade de vida SF-36, que já existe a versão com 12 itens, inclusive validada em português do Brasil 4.

Nessa mesma linha, a CASP-19 já apresenta a sua versão com 12 itens, já realizada na população espanhola e na etíope sem perda das propriedades psicométricas 5,6, cuja validação será realizada em português pelo nosso grupo.
1 Universidade de

Pernambuco, Recife, Brasil.

2 Universidade Federal do

Rio de Janeiro, Rio de Janeiro,

Brasil.

Correspondência

F. Lima

Universidade de Pernambuco.

Avenida Agamenon

Magalhães s/n Recife, PE

50100-010, Brasil.

fabia.lima@hotmail.com 
(4) A validação de Neri et al. ${ }^{1}$ conta com algumas limitações, além das já listadas. Afirmam que a nova versão da CASP-19 é eficaz para avaliar a qualidade de vida percebida em indivíduos de 55 anos ou mais, quando a amostra foi feita por conveniência, sendo constituída por $86 \%$ de mulheres e $22 \%$ tinham de 44-59 anos, ou seja, adultos e não apenas idosos, o que pode limitar essas conclusões. Outra limitação é a de que foi composta por indivíduos com boa escolaridade (pertencentes a Universidades da Terceira Idade e cursos de educação em saúde), tendo sido excluídos os analfabetos, o que certamente limitaria a aplicação desta versão para uma população idosa não letrada.

No Brasil, de acordo com o Instituto Brasileiro de Geografia e Estatística (IBGE) 7, por intermédio da Pesquisa Nacional por Amostra de Domicílios Contínua (PNAD Contínua) referente ao ano de 2016, 20,4\% dos idosos eram analfabetos. Desse modo, acreditamos que a validação de Neri et al. é válida para um grupo especifico de pessoas acima de 44 anos e com boa escolaridade, e não se aplica à população dos idosos brasileiros, como a de Lima et al. 2, que avaliou apenas maiores de 65 anos e pertencentes às classes sociais alta, média e baixa, compostas também por analfabetos.

Agradecemos as críticas de Neri et al., que são sempre importantes para o crescimento acadêmico, e desejamos que mais estudos sejam feitos no Brasil com a escala CASP, a fim de que possamos ter mais comparações da população idosa brasileira, europeia e dos Estados Unidos. 


\section{Colaboradores}

F. Lima, K. Patribu, J. Laks e M. S. Campos colaboraram na concepção, redação e revisão do texto.

\section{Informações adicionais}

ORCID: Fabia Lima (0000-0001-9992-6556); Kátia Patribu (0000-0002-8861-0238); Jerson Laks (0000-0002-5129-9087); Marilia Siqueira Campos (0000-0002-6834-6003).

\section{Referências}

1. Neri AL, Borim FSA, Batistoni SST, Cachioni M, Rabelo DF, Fontes AP, et al. Nova validação semântico-cultural e estudo psicométrico da CASP-19 em adultos e idosos brasileiros. Cad Saúde Pública 2018; 34:e00181417.

2. Lima FM, Hyde M, Chungkham HS, Correia C, Siqueira Campos A, Campos M, et al. Quality of life amongst older Brazilians: a cross-cultural validation of the CASP-19 into BrazilianPortuguese. PLoS One 2014; 9:e94289.

3. Beaton DE, Bombardier C, Guillemin F, Ferraz MB. Guidelines for the process of cross-cultural adaptation of self-report measures. Spine 2000; 25:3186-91.

4. Silveira MF, Almeida JC, Freire RS, Haikal DS, Martins AE. Propriedades psicométricas do instrumento de avaliação da qualidade de vida: 12-item health survey (SF-12). Ciênc Saúde Colet 2013; 18:1923-31.

5. Hamren K, Chungkham HS, Hyde M. Religion, spiritualy, social support and quality of life: measurement and predictors CASP-12 (v2) amongst older Ethiopians living in Addis Ababa. Aging Ment Health 2015; 19:610-21.

6. Pérez-Rojo G, Martín N, Noriega C, López J. Psychometric properties of the CASP-12 in a Spanish older community dwelling sample. Aging Ment Health 2018; 22:700-8.

7. Instituto Brasileiro de Geografia e Estatística. Pesquisa Nacional por Amostra de Domicílios Contínua. Educação 2017. Rio de Janeiro: Instituto Brasileiro de Geografia e Estatística; 2017. 